\title{
Automated Monitoring of Foraging Behaviour in Free Ranging Sheep Grazing a Biodiverse Pasture
}

\author{
A. Mason ${ }^{1}$ and J. Sneddon ${ }^{2}$ \\ ${ }^{1}$ School of Built Environment, Liverpool John Moores University, Liverpool, UK \\ ${ }^{2}$ School of Natural Sciences and Psychology, Liverpool John Moores University, Liverpool, UK. \\ a.mason1@ljmu.ac.uk
}

\begin{abstract}
Little is currently known about the foraging behavior of free ranging animals, particularly in biodiverse pastures. This is despite the suggestion of recent work that animals grazing on such pastures tend to produce better quality meat. This paper presents a bespoke Wireless Sensor Network system designed to be mounted on grazing animals and collect movement information which is then coded with reference to human observations. In doing this it has been possible to calibrate the bespoke system such that, in realtime, the system can be used to deduce animal behavior (e.g. resting, grazing, foraging, etc.) remotely. When coupled with future GPS-free positional information, this system will provide valuable information for the UK agricultural industry, in addition to overcoming the challenges faced by many commercial systems which rely on energy intensive GPS technology.
\end{abstract}

Keywords- Behaviour monitoring, wireless sensor networks, tracking, location, accelerometer, animal grazing, biodiverse pasture.

\section{INTRODUCTION}

There is a movement in the UK livestock production systems to enhance and optimize lower input grass based farming systems [1], to eat locally, and to use sustainable local resources. The Regional Land Use project 'Eating biodiversity' provided scientific evidence that meat from animals that ate pasture rich in biodiversity was of superior quality $[1,2]$.

The work reported in this paper is part of a larger and ongoing scheme of work which focuses on two elements: 1) the ability to use sensor technology to monitor the grazing behavior of animals in real-time and;2) the positioning of animals as they move around their grazing area.

The technology being developed and currently in use for the work is based upon Wireless Sensor Network (WSN) technology (or, in this context, Radio Frequency Identification [RFID]). This technology was originally developed at Liverpool John Moores University (LJMU) for the purpose of tracking commercial goods [3, 4], namely gas cylinders, due to the robustness of the ad hoc networking approach available to WSN systems in harsh environments.

The application here with grazing animals presents new challenges, in particular the greater range required by sensor nodes; while gas cylinders tend to be tightly packed in storage crates, free ranging sheep do not. However the principle of ad hoc networking (as illustrated in Figure 1) afforded by sensor networks will provide significant benefit in this application.

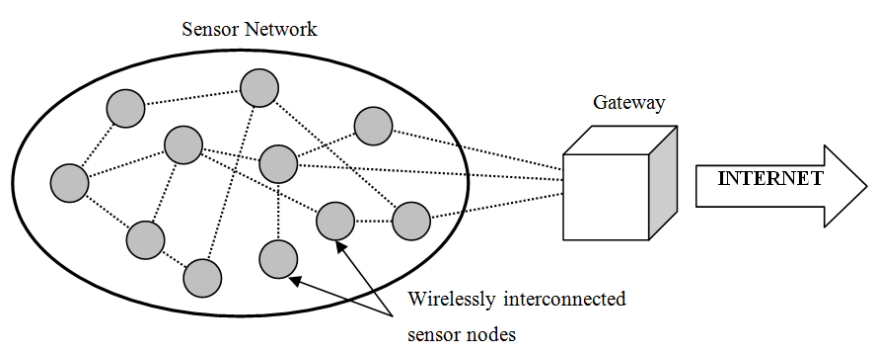

Figure 1. The ad hoc nature of WSN systems, which enables long-range network connectivity when physical radio transmission range between individual nodes is small.

Many prior animal tagging systems which provide realtime data (ignoring systems which only provide identification services) are based on GPS technology [5-7]. While this has been proven to give information regarding the behavior of grazing animals, it suffers from the following issues: 1) poor battery life; 2) low resolution positioning; and 3) failure in poor weather or when animals seek shelter on hotter days. All of these cause issue for researchers interested in identifying precisely what an animal is eating over the course of a grazing season, which in the UK normally runs from May until September. Poor battery life leads to high maintenance costs, which are prohibitive for widespread use in a commercial setting and provide challenges in ensuring data validity and completeness in the research domain. In addition, researchers often find the low resolution of GPS technology (i.e. with accuracy tending to be no better than 3 meters) to be insufficient when attempting to identify the particular areas (i.e. within centimeters) in which animals are grazing. This is exacerbated in the context of this work since in bio-diverse pastures the type of grass can vary enormously, even over an area of a few square meters.

Thus, this work demonstrated the successful use of WSN technology to monitor the behavior of animals in addition to providing positional information which will allow researchers 
to accurately assess the state of animals (i.e. grazing, resting, ruminating, etc.) and also the location in which they undertake such activities. While others have used WSN systems for monitoring animal activity and behavior [8-10], the novel aspect of this work is two-fold: 1) the application of such technology on free-ranging animals in bio-diverse pastures and 2) the future implementation of GPS-free positioning. Therefore, this paper focuses on initial work to establish the operation of a bespoke WSN system, showing the response of on-board sensors to changes in animal behavior. Forthcoming publications will tackle the issue of the system providing location services. In achieving these goals, the work will provide valuable information to underpin wide-scale UK agricultural objectives, including:

- Improvement of animal nutrition and growth through better feeding methods, since the information obtained will allow the qualification and quantification of foraging behavior under natural grazing conditions. This information can be used to improve grasslands for animals and biodiversity.

- Development of better integrated systems for beef and sheep production, since to date there is no detailed data related to the cost benefits of natural foraging behavior to the degree of detail that would be obtained via real-time technologies, such as that proposed here, and to be described later in this paper.

- Provision of practical responses to climate change mitigation and adaptation, as sustainable management of pasture resources is required for the prevention of soil erosion.

The remainder of this paper discusses; 1) the WSN system currently in use and on-going development as the project progresses; 2) the methodology undertaken for acquiring preliminary data from sheep in a small (approx. $100 \mathrm{~m}^{2}$ ) site; 3) results from this preliminary work and finally; 4) the key findings so far along with the future direction of the research.

\section{THE WIRELESS SENSOR NETWORK SySTEM}

The WSN system constructed for this work is based upon the Texas Instruments CC2431 system-on-chip (SoC) microprocessor. This provides both processing capability, via an 8051 based core, and radio frequency transmission at the ISM $2.4 \mathrm{GHz}$ band. The CC2431 microprocessor used is embedded in a Texas Instruments CC243x evaluation module (EM) for simplicity, and we have developed custom software in addition to a custom breakout board for the EM. This customization has allowed us to develop a system which incorporates an accelerometer sensor, while being small enough to mount on a sheep without any noticeable change in the animal behavior. Figure 2(a) shows a block diagram overview of the system and Figure 2(b) shows the sensors as constructed and packaged prior to mounting on an animal.

The sensor itself was envisaged to be placed on or near the head/neck of the animal so as to be able to detect head movement. This is vital, since the animals head orientation is an important indicator of whether the animal is grazing or not. Detection of this movement is provided by a 3-axis accelerometer (Freescale Semiconductor MMA7361L). As the system is developed further, linking the instances when an animal is grazing and positional data will provide key information regarding what the animal is eating. Furthermore, the accelerometer information can be used in other ways, for example to determine whether an animal is sleeping or simply resting and/or ruminating. This may yield yet further interesting information (e.g. animal health status and change in normal behavioral patterns), or on the other hand, it may prove useful in aggregating the large amounts of data that can be easily collected using a continuous real-time WSN system.

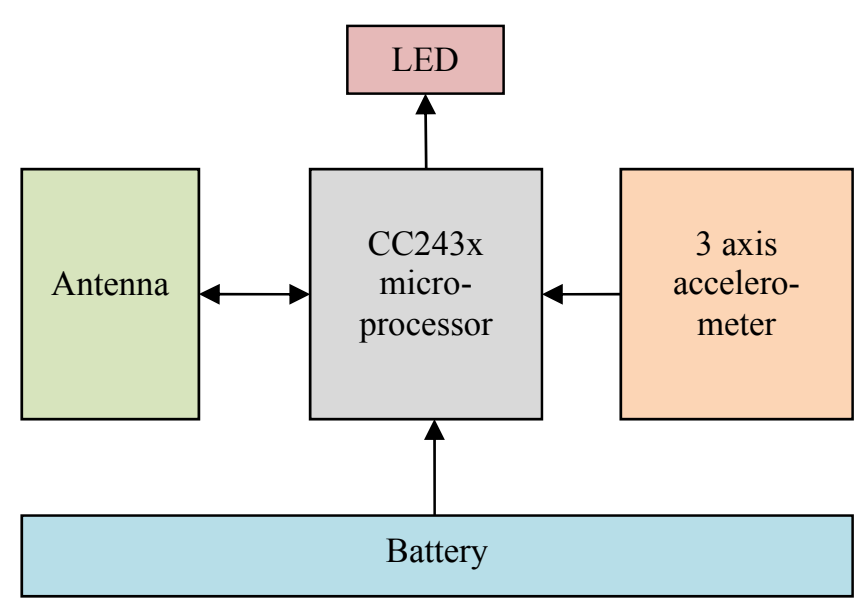

(a)

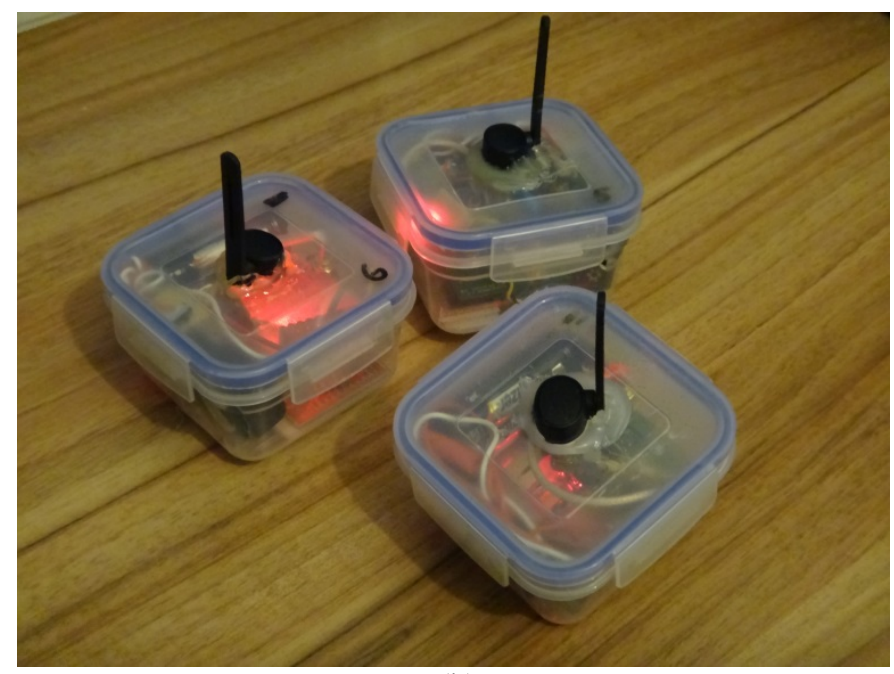

(b)

Figure 2. (a) A block diagram overview of the sensor system showing all major components and (b) the physical sensors constructed prior to being installed on a sheep halter for field use. 
The sensor is powered using four 'AAA' type batteries, with a low drop-out voltage regulator then converting this $6 \mathrm{~V}$ to a $3.3 \mathrm{~V}$ power source for the CC243xEM as well as sensor components. This combination is utilized to minimize sensor drift as the batteries become worn with use. Currently the lifetime of the individual node with a duty cycle of $15 \%$ is approx. 5 days continuous operation. Although the CC243x does support low power operation which would extend this lifetime significantly, it is likely that we shall replace this microprocessor with a newer variant in the near future, and therefore we wish to minimize any unnecessary duplication of effort.

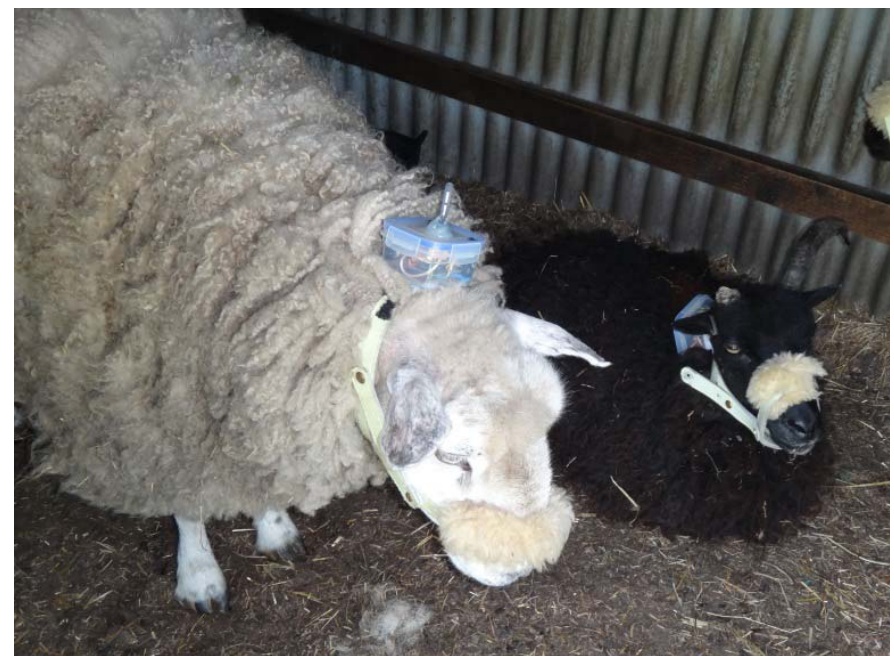

(a)

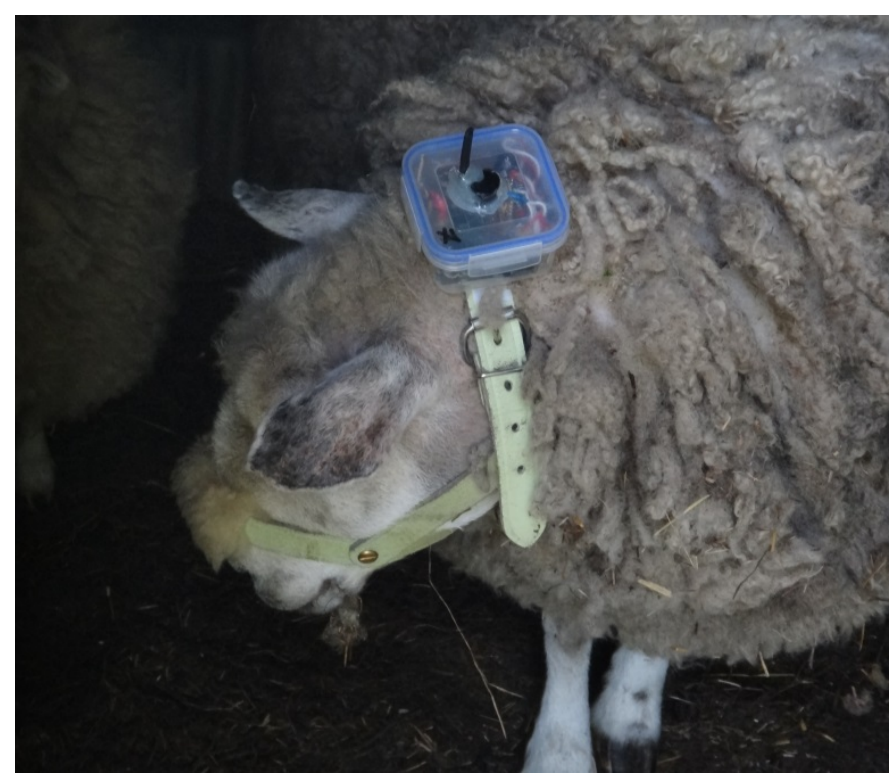

(b)

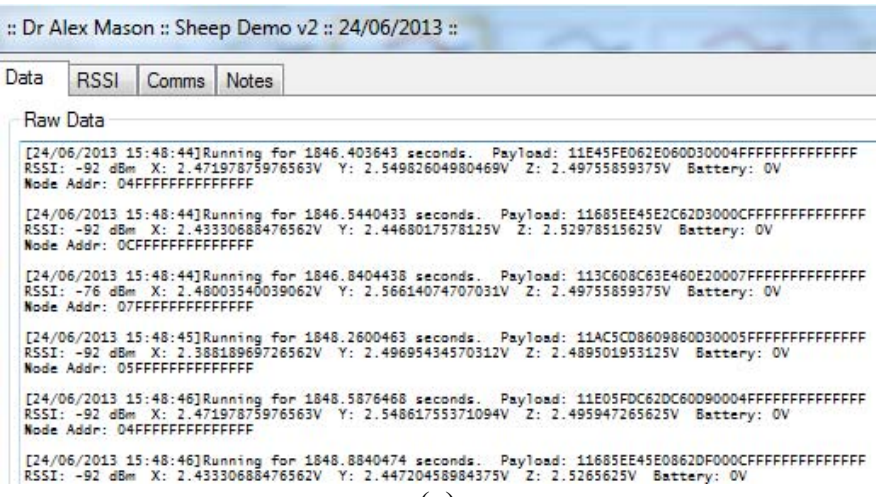

(c)

Figure 3. (a) The sensors mounted on two different types of sheep, (b) a close-up of the mounted sensor and (c) the bespoke data logging software interface.

The sensor system and associated electronics are housed inside a water-tight (non-IP rated) container with external dimensions of $80 \times 80 \times 50 \mathrm{~mm}(w \times l \times h)$. This container has a hole drilled in the base to allow for a rivet which fixes it to a sheep halter with Velcro providing some addition stability. In the future it is likely the system will be integrated into a robust collar which can be worn permanently by animals. The sensors mounted on sheep are shown in Figure 3(a) and Figure 3(b); in the former image one can see the different breeds of sheep used in this work, and in the latter it is possible to see the sensor as attached to the animal halter.

Once operational, as indicated via a simple blinking LED, the sensor nodes communicate accelerometer data every 2 seconds, and a base station connected to a PC is used to collect this data. A bespoke C\# application [see Figure 3(c)] logs all received data to CSV for latter interpretation.

\section{METHOD}

Four mature ewes were supplied at OS location 333781,371970 Shotwick, Cheshire UK. Figure 4 shows an overview of the testing site, and Table 1 provides information regarding the attributes of the sheep used. Each sheep was fitted with a chromium tanned leather halter (Kamer Ltd), which had the sensor system, described in Section II, attached.

Foraging behavior in the sheep was examined on this biodiverse mature pasture during June and July 2013. The fields contain clover-rye grass dominated pastures with additional red fescue, Yorkshire fog, timothy, sweet vernal grass and meadow grass mixtures ( $n=60$ quadrats/field). The fields had not received artificial fertilizer for at least 20 years and had been lightly grazed by sheep ( $\mathrm{n}=3$ or 4 ) for the last 10 years. Field 1 included a chicken run and field 2 a paddling pool and 5 fruit trees. 


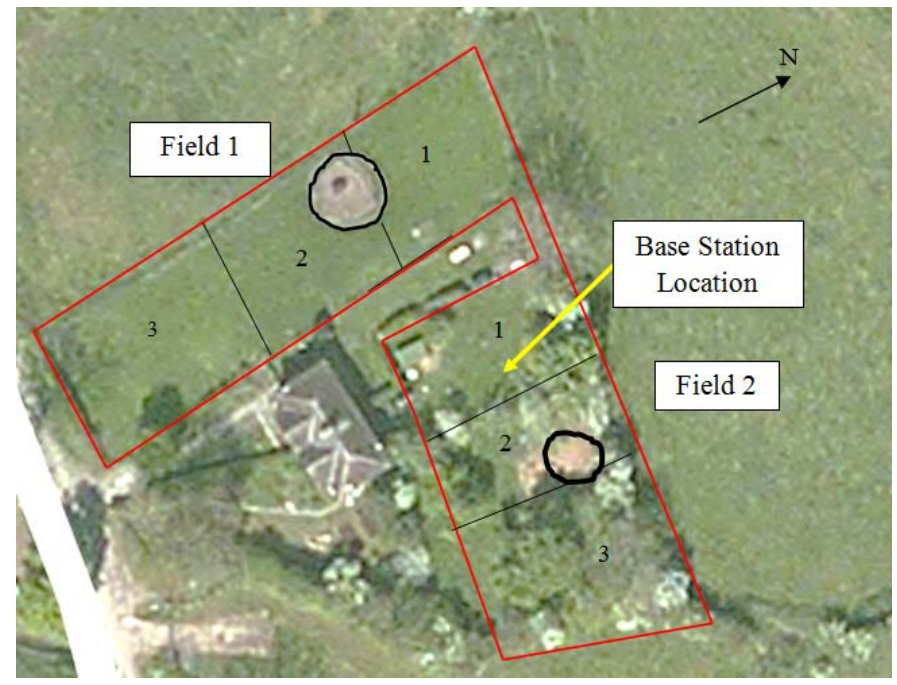

Figure 4. Overview of testing location, showing the two fields utilized during testing, with each field being joined by a narrow pathway. The total area of the fields combined is approx. $100 \mathrm{~m}^{2}$.

Table 1. Attributes of sheep $(n=4)$ in the small flock used in this study.

\begin{tabular}{|l|c|c|}
\hline Species & $\begin{array}{c}\text { Age } \\
\text { (Years) }\end{array}$ & Mass (kg)* \\
\hline Texel & 9 & 74.1 \\
Welsh Balwyn & 8 & 34.9 \\
Hebridean & - & - \\
Hebridean & - & - \\
*based on body surface area determined by a tiling method.
\end{tabular}

The base station was situated at the point indicated in Figure 4. A device-to-base station range of $60 \mathrm{~m}$ was found to be achievable and so this location gave adequate range in the worst case that the sheep became separated; in practice this rarely happened. No notable interference from trees and other obstacles within the fields were observed; received signal strength data was collected in order to confirm this. Accelerometer data was captured at 2 second intervals, with human logging of sheep activity also taking place so that the data acquired and actual sheep behavior could be matched offline for coding and calibration purposes. Analysis of this data is presented in Section IV.

\section{RESUlts AND DisCUSSION}

The use of a 3-axisaccelerometer for determining foraging behaviors in a range of animals has been reviewed by Shephard et al [11]. Principal Component Analysis (PCA) was employed to assess the accuracy of assigned behavioral codes to groupings given based upon observation of the sheep over the course of this experimental work. Behavior codes, numbers between 0 and 4 inclusive, were assigned as follows:
(0) Placing halter on sheep, typically at the beginning of an observation session;

(1) Standing but not eating/foraging;

(2) Grazing;

(3) Browsing (or foraging);

(4) Laying.

Table 2 shows examples how these behavioral codes were developed over the course of multiple days using data from the various sheep. The Y-axis data was used to code data (1 or 2) via manual scanning of a time-based scatter graph or by scanning the values in the data set. This approach did not appear to be any more subjective than the use of manual observation for coding of behaviors.

Table 2. Coding of accelerometer data based upon manual observations. Each sheep is identified by an ID number (as shown) transmitted from the sensor devices, and the data below represents a small sample of the total collected.

\begin{tabular}{|c|c|c|c|c|}
\hline ID & Breed & Date & $\begin{array}{c}\text { \% } \\
\text { accuracy** }\end{array}$ & $\begin{array}{c}\text { Deduced } \\
\text { behavior codes }\end{array}$ \\
\hline 7 & Texel & 24.06 .13 & 87.1 & 1,2 \\
\hline 5 & Hebridean & 25.06 .13 & 59.3 & $0,1,2,3,4$ \\
\hline 7 & Texel & 25.06 .13 & 91.9 & $1,2,3$ \\
\hline 4 & Hebridean & 28.06 .13 & 70.8 & 1,2 \\
\hline 5 & Hebridean & 28.06 .13 & 83.9 & 1,2 \\
\hline
\end{tabular}

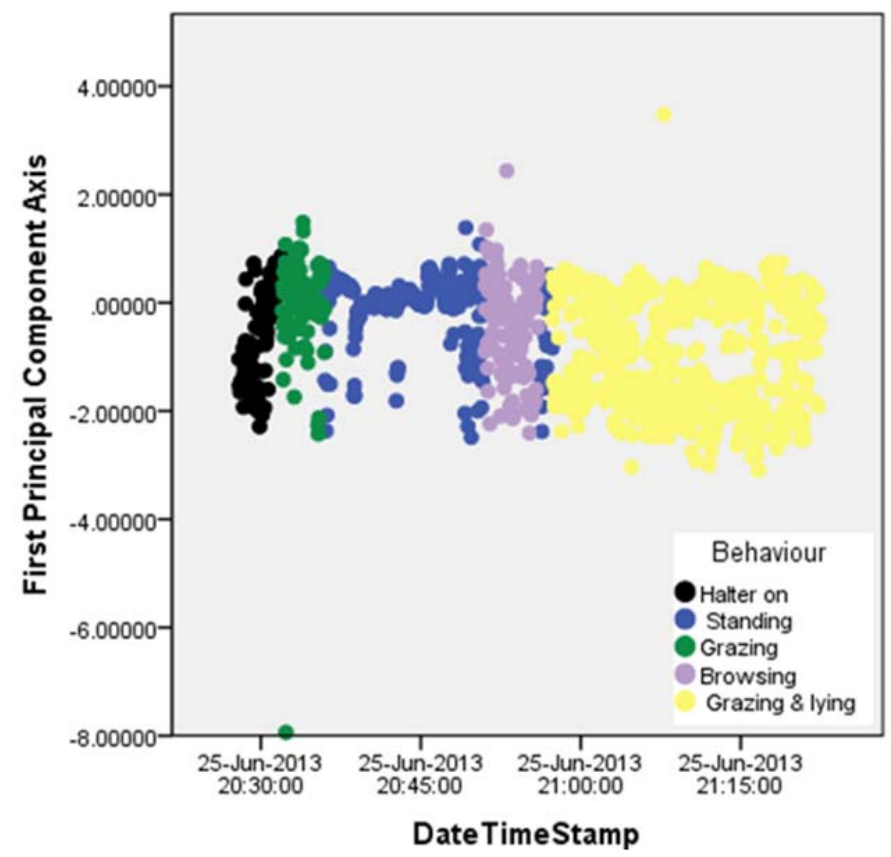

Figure 5. Data from a Hebridean sheep (25.06.13), with behaviors depicted by changes in accelerometer output due to sheep movement. Coding schemes are clearly highlighted here to show periods were the sheep is: 1) having a halter 
fitted; 2) standing idle; 3) grazing; 4) browsing and 5) lying while grazing.

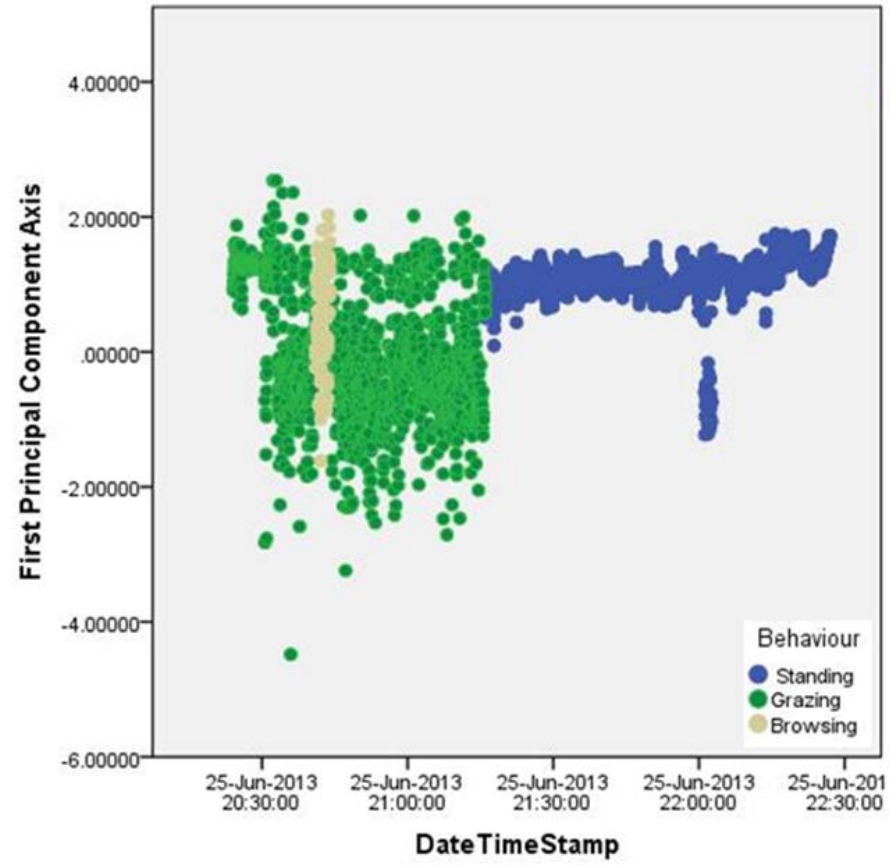

Figure 6. From Table 2 it is shown that behaviors 1 (standing) and 2 (grazing) are most commonly observed. The data shown in the above chart give a clear distinction between grazing activity and idle, i.e. when the sheep is not eating, which could be readily utilized for real-time determination of animal behavior.

Figure 5 shows data from a sheep where the entire range of behavioral codes were observed. The chart clearly separates the different activities through color, with it becoming apparent that changing activity shows marked changes in accelerometer response at discrete time internals as well as over defined ranges in time.

Figure 6 deals predominantly with the cases of grazing and standing; these behaviors were observed across all days with the sheep and so there is significant confidence that the system is able to determine when grazing is taking place. The difference between the areas highlighted in green (grazing) and blue (standing) in Figure 6 support this, as do the high accuracy levels for the days in Table 2 where only behavioral codes 1 and 2 are deduced. While further data collection and observation may yield more information on subtle aspects of animal behavior, determination of when an animal is eating is of great importance to this work. As we progress and add positional information, the system should allow determination of what was eaten, thus providing vital data to inform future practice in the utilization of biodiverse pastures for animal grazing.

\section{CONCLUSION}

This paper presents initial findings from an on-going project to assess animal foraging behavior when grazing biodiverse pastures. A prototype WSN system has been developed and implemented for use in the field, and has yielded promising initial data which shows that one could, remotely and in realtime, determine the current behavior of an animal. In particular we have shown significant variation in the output of an accelerometer sensor when an animal is grazing versus when it is simply standing.

Future work will seek to link this information to positional data in order that researchers can quickly assess what animals are choosing to eat in a biodiverse pasture. This will inform future best practice in the agricultural industry by the improving animal nutrition and developing better integrated systems for animal production. Knowledge of where animals are grazing could also allow deduction of resultant contamination (i.e. through urination) in particular areas. This means that farmers can take timely remedial action, including measures such as rotation of animal grazing or land treatment. In addition, further work will seek to employ a small animal mounted video camera in order to provide a recorded data set against which to deduce codes for the accelerometer output. It is hoped that this will allow for finer levels of coding in order to accurately deduce subtle changes in animal behavior.

\section{ACKNOWLEDGEMENT}

The authors would like to acknowledge the support of The Organisation for the English Beef and Sheep Industry (EBLEX), project reference 73602 .

\section{REFERENCES}

[1] H. Buller, "Eating Biodiversity: An Investigation of the Links between Quality Food Production and Biodiversity Protection: Full Research Report," ESRC, Swindon2007.

[2] B. Smith and S. Everet, "Food Farming and Conservation, report on a seminar in October 2009 held at Fordingbridge by Flora Locale and Agricultural Ecology Special Interest Group of the British Ecological Society," 2009.

[3] A. Mason, A. Shaw, and A. Al-Shamma'a, "Peer-to-peer inventory management of returnable transport items: A design science approach," Computers in Industry, vol. 63, pp. 265-274, 2012.

[4] A. Mason, A. I. Al-Shamma'a, and A. Shaw, "Inventory Management in The Packaged Gas Industry Using Wireless Sensor Networks," in Advances in Wireless Sensors and Sensor Networks. vol. 64, Subhas Chandra Mukhopadhyay and H. Leung, Eds., 1st ed: Springer, 2010, pp. 75-100.

[5] C. Umstatter, A. Waterhouse, and J. P. Holland, "An automated sensor-based method of simple behavioural classification of sheep in extensive systems," Comput. Electron. Agric., vol. 64, pp. 19-26, 2008.

[6] K. Betteridge, C. Hoogendoorn, D. Costall, M. Carter, and W. Griffiths, "Sensors for detecting and logging spatial distribution of urine patches of grazing female sheep and cattle," Comp Electr Agric, vol. 73, pp. 8-8, 2010 . 
[7] H. L. Perotto-Baldivieso, S. M. Cooper, A. F. Cibils, M. Figueroa-Pagán, K. Udaeta, and C. M. Black-Rubio, "Detecting autocorrelation problems from GPS collar data in livestock studies," Applied Animal Behaviour Science, vol. 136, pp. 117-125, 2012.

[8] E. S. Nadimi, R. N. Jørgensen, V. Blanes-Vidal, and S. Christensen, "Monitoring and classifying animal behavior using ZigBee-based mobile ad hoc wireless sensor networks and artificial neural networks," Computers and Electronics in Agriculture, vol. 82, pp. 44-54, 2012.

[9] E. S. Nadimi, H. T. Søgaard, T. Bak, and F. W. Oudshoorn, "ZigBee-based wireless sensor networks for monitoring animal presence and pasture time in a strip of new grass," Computers and Electronics in Agriculture, vol. 61, pp. 79-87, 2008.

[10] E. S. Nadimi, V. Blanes-Vidal, R. N. Jørgensen, and S. Christensen, "Energy generation for an ad hoc wireless sensor network-based monitoring system using animal head movement," Computers and Electronics in Agriculture, vol. 75, pp. 238-242, 2011.

[11] E. Shepard, R. Wilson, F. Quintana, A. GÃmez Laich, N. Liebsch, D. Albareda, L. Halsey, A. Gleiss, D. Morgan, A. Myers, C. Newman, and D. Macdonald, "Identification of animal movement patterns using triaxial accelerometry," Endangered Species Research, vol. 10, pp. 47-60, March 31, 20082008. 